

\title{
SPATIAL EXPANSION OF HOT ELECTRON-HOLE PLASMA AT HIGH DENSITY IN CdSe
}

A. Cornet, M. Pugnet, J. Collet, Thierry Amand, M. Brousseau

\section{To cite this version:}

A. Cornet, M. Pugnet, J. Collet, Thierry Amand, M. Brousseau. SPATIAL EXPANSION OF HOT ELECTRON-HOLE PLASMA AT HIGH DENSITY IN CdSe. Journal de Physique Colloques, 1981, 42 (C7), pp.C7-471-C7-476. 10.1051/jphyscol:1981757 . jpa-00221694

\section{HAL Id: jpa-00221694 https://hal.science/jpa-00221694}

Submitted on 1 Jan 1981

HAL is a multi-disciplinary open access archive for the deposit and dissemination of scientific research documents, whether they are published or not. The documents may come from teaching and research institutions in France or abroad, or from public or private research centers.
L'archive ouverte pluridisciplinaire HAL, est destinée au dépôt et à la diffusion de documents scientifiques de niveau recherche, publiés ou non, émanant des établissements d'enseignement et de recherche français ou étrangers, des laboratoires publics ou privés. 
JOURNAL DE PHYSIQUE

ColZoque C7, supplément au $n^{\circ} 10$, Tome 42 , octobre 1981

page $\mathrm{c} 7-471$

\title{
SPATIAL EXPANSION OF HOT ELECTRON-HOLE PLASMA AT HIGH DENSITY IN CASE
}

\author{
A. Cornet, M. Pugnet, J. Collet, T. Amand and M. Brousseau
}

Laboratoire de Physique des Sozides associé au C.N.R.S., I.N.S.A., Avenue de Rangueil, 31077 Touzouse Cedex, France

\begin{abstract}
Résumé : Nous présentons la première étude expérimentale du transitoire à T'échel le picoseconde de l'expansion d'un plasma chaud et très dense dans cdSe (en platelet) fortement excité par une impulsion d'un laser YAG ( $\mathrm{Nd}^{3+}$ ) à modes synchronisês de durée 30 ps. Nous montrons qu'au-dessus d'une densité critique, le plasma explose à une vitesse qui est supérieure de deux ordres de grandeur à la valeur de la vitesse de diffusion à l'équilibre thermodynamique. Nous utilisons nos calculs théoriques antérieurs pour analyser les résultats.

Abstract : We report here the first experimental study of time resolved expansion in the picosecond time scale of hot electron-hole plasma at very high density generated in CdSe platelets strongly excited by a mode locked Yag laser pulse of duration $30 \mathrm{ps}$. We show that above a critical density, the plasma expands at a velocity which is two orders of magnitude greater than the thermodynamic equilibrium diffusion velocity value. We use our previous theoretical work for the analysis of the results.
\end{abstract}

Introduction. - In direct gap semiconductors the electron hole plasma luminescence can be used as a probe for testing the presence of the plasma in a defined region of a sample; we apply here this idea to the high density plasma in CdSe at low temperature, using appropriate experimental conditions.

1. Experimental set up. - A complete description of our experimental apparatus has been previousiy reported [1], [2], [3]. In the present experiments, a picosecond puTse (30 ps) is used to excite the sample (Fig. 1). The intensity of the pulse, measured by a photodiode, is introduced into a two levels discriminator which defines a narrow window for pulse energy. When the energy of the excitation pulse falls within this energy window, the two levels discriminator authorizes the processing of the experimental data by multichannel computer. Two photons absorption at $1.064 \mu \mathrm{m}$ gives rise to homogeneous excitation in CdSe platelets, according to recent measurements [4]. The luminescence of the sample is focalised into a Kerr cell which transmits the incident light only when it is excited by the pulse at $1.064 \mu \mathrm{m}$, that means during $30 \mathrm{ps,}$ and so are obtained the time resolved spectra. The transmitted luminescence enters a monochromator and is finally detected by an optical multichannel analyser monitored by the energy window discriminator of the excitation.

The sample is a platelet, grown in vapor phase, of typical dimensions $1 \times 1 \times 0.03 \mathrm{~mm}^{3}$. We have choosen a thin sample for two reasons:

- first, we get an homogeneous excitation; the inhomogeneities in the excitation are less than $10 \%$ between the front surface and back surface of the platelet; 
- Secondly, the stimulation is low in the detector direction (Y) (see Fig. 2) and we shall neglect it in our calculations.

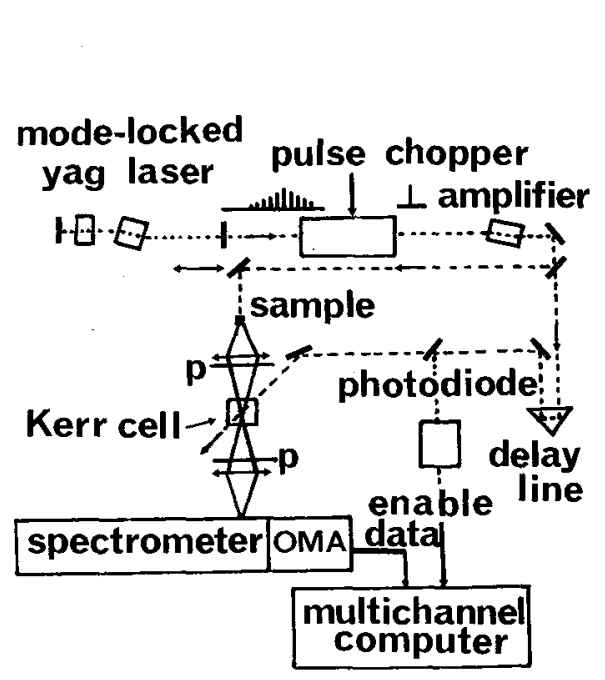

Fig. 1

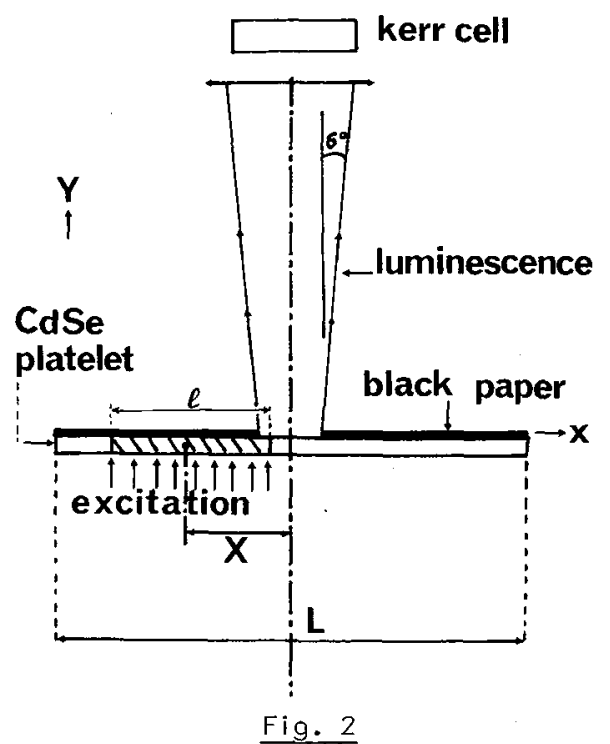

Fig. 2

The excited region is a parallelipipedic volume $\left(1 \times 0.3 \times 0.03 \mathrm{~mm}^{3}\right)$ (see Fig. 2$)$. The luminescence emerges through a slit (width $100 \mu \mathrm{m}$ ) in a black paper which covers all the back surface of the sample, but, only are detected the photons which spring in a solid angle of $\pi / 100$ steradian, in order to increase the spatial definition of the luminescent plasma. The principle of the experiment is to get a time and wavelength resolution of the luminescence which springs from the back side slit for various positions $X$ of the excited region (Fig. 2) so that we obtain the space and time resolved luminescence spectrum of the sample. The CdSe platelet is stuck on a copper cold finger in an optical cryostat. When the incident power is in the range $100 \mathrm{MW} / \mathrm{cm}^{2}<P<1.5 \mathrm{GW} / \mathrm{cm}^{2}$, the plasma density is given by $n \simeq \beta P^{2}(1+\beta P e)^{-1}(2 h \nu)^{-1}(1)$ where $\beta$ is the two photons absorption coefficient; $\beta \simeq 0.03 \mathrm{~cm} / \mathrm{MW}$ for $\lambda=1.064 \mu \mathrm{m}$ $[4]$, e is the sample thickness, and $h \mathcal{\nu}$ is the excitation photon energy. In this work, the plasma density is always calculated using the formula (1) ; when the incident power increases, the exciton line (present at low excitation) disappears at a density $n=1.5 \times 10^{17} \mathrm{~cm}^{-3}$, derived in this way, which is in good agreement with previous experimental determination for the Mott density [5].

When the incident power $P$ is lower than $1.5 \mathrm{GW} / \mathrm{cm}^{2}$, the lattice heating due to 7 aser pulse is always lower than $15 \mathrm{~K}$ [3]. The experimerits are performed successively at $T_{L} \simeq(10-20 \mathrm{~K}), T_{L} \simeq(80-90 \mathrm{~K})$ and $T_{L} \simeq 300 \mathrm{~K}$.

2. Experimental results and analysis. - A. Experimental results.- The figure 3 shows two time resolved 1 uminescence spectra due to recombination of electron-hole pairs in the plasma, assisted by the emission of an LO phonon. In the range $300 \mathrm{MW} / \mathrm{cm}^{2}<\mathrm{P}<1.5 \mathrm{GW} / \mathrm{cm}^{2}$, the band gap reduction induces a shift of the luminescence 


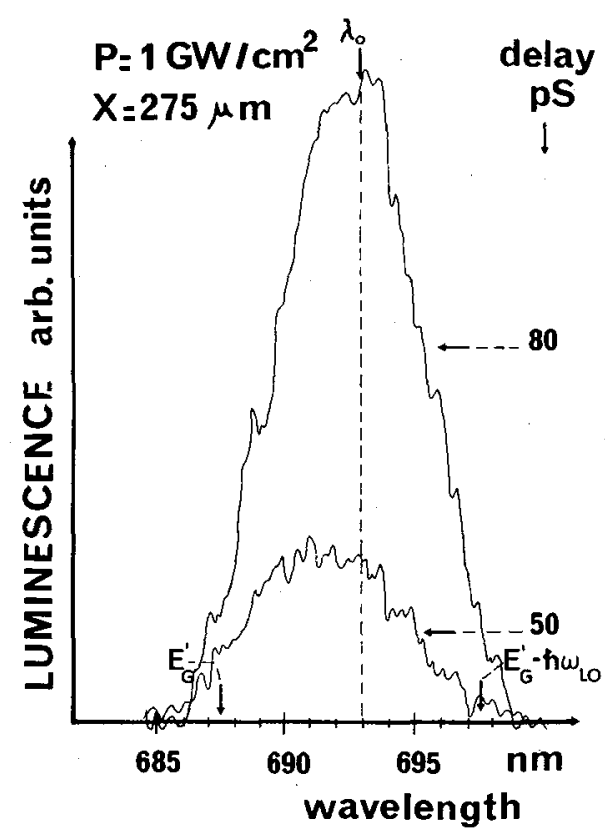

Fig. 3: Time resolved LO-phonon assisted plasma lumi nescence originating from a non-laser excited region $(X=275 \mu \mathrm{m})$.

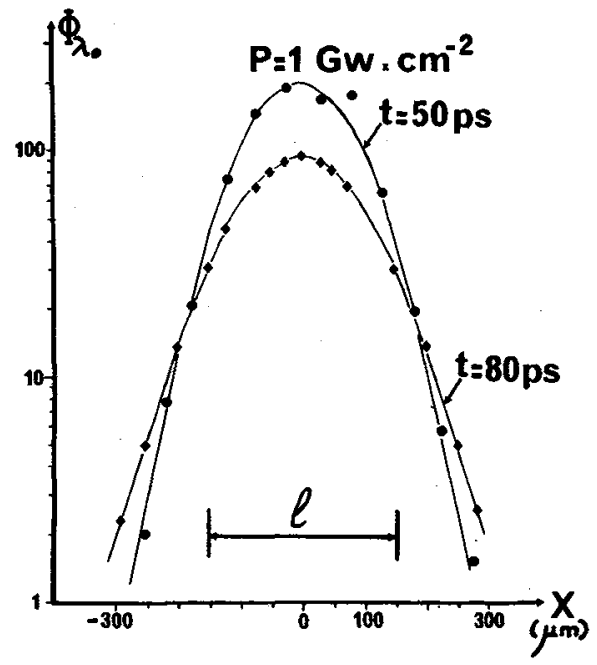

Fig. 4 : Two post-excitation distributions of time resolved luminescence. - : Theoretical fit with (8). but the process is always the same. In a detailed analysis [2] we have shown that at high plasma temperature, the low energy edge is the renormalized band gap $E_{G}^{\prime}$ minus the longitudinal optic phonon energy $\hbar \omega_{10}$ and the upper edge is approximately the renormalized band gap itseif. Consequently, this luminescence is an evidence for the presence (at the point $X$ ) of high density electron hole plasma. The time resolved spatial distribution of the detected Tuminescence is strongly dependent on the excitation : under a threshold $\mathrm{Pe}$, the light springs only from the initially excited region, while above this critical value $P e$, the light emerges from the whole sample.

Fig. 4 shows the luminescence $\Phi_{\lambda_{0}}$ at a given wavelength in the plasma band versus $X$ for an incident-time averaged-power $P=1 \mathrm{GW} / \mathrm{cm}^{2}$ at two times $t_{1}=50 \mathrm{ps}$ and $t_{2}=80$ ps. The time origin is defined as the luminescence threshold. When $P$ is grea-

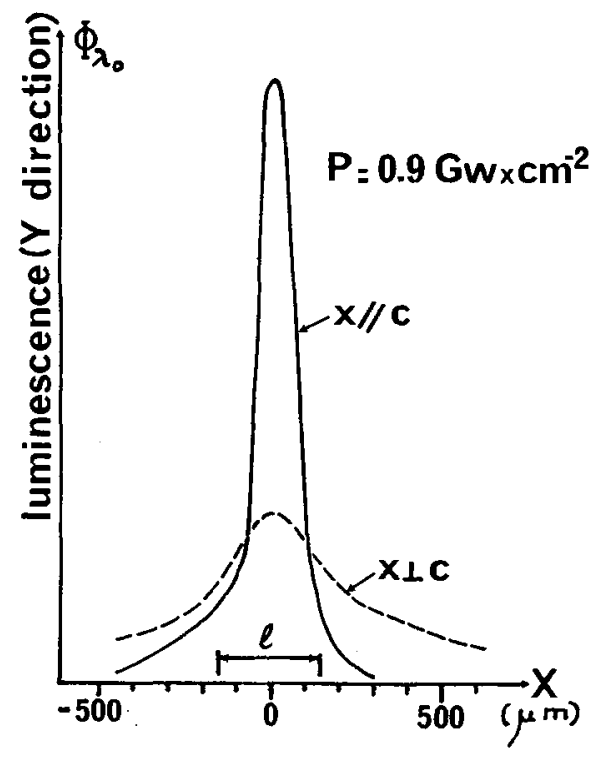

Fig. 5: Time integrated Iuminescence for two relative directions of propagation with respect to the c-axis (the effect is similar for time resolved I umi nescence). 
ter than a critical value $\mathrm{Pe}$ (which is dependent on the relative direction $x$ with respect to the c-axis) the light emerges from the whole sample before the end of the laser pulse. We find $\operatorname{Pe}(x / / \mathrm{c}) \simeq 0.9 \mathrm{GW} / \mathrm{cm}^{2}$ and $\operatorname{Pe}(x \perp c) \simeq 0.8 \mathrm{GW} / \mathrm{cm}^{2}$.

A11 these results are similar when the experiment is performed at $T_{L} \sim 10 \mathrm{~K}$ and $T_{L} \sim 80 \mathrm{~K}$; on the contrary, we cannot detect any luminescence outs $i d e$ of the excited region when $T_{L} \sim 300 \mathrm{~K}$.

B. Analys is. - For a quantitative analysis, a diffusion model is used, in which the diffusivity $D$ is only a parameter; the fit of the two curves $\left(t_{1}=50 \mathrm{ps}\right)$ and $\left(t_{2}=80\right.$ ps $)$ in Fig. 4 with this model, allows us to give an order of magnitude for $D$. The spatial plasma density $n(x)$ in the $x$ direction depends on the photon density (1uminescence) distribution within the sample via the stimulated emission process [6] ; this is a very difficult problem because of the gain saturation of stimulated luminescence. E.0. Göbel and a1. [6] proposed a self-consistent model in which $n(x)$ is a symetric bell-shaped function.

Here, for simplicity, we assume :

$$
n(x, t=0)=N \exp -\frac{4 \log 2}{a^{2}} x^{2}
$$

where a and $\mathrm{N}$ are parameters.

The diffusion is governed by :

$$
\frac{\partial n}{\partial t}(x, t)=-\frac{n(x, t)}{\tau}+D \frac{\partial^{2} n(x, t)}{\partial x^{2}}
$$

where $D$ and $\tau$ are the "ambipolar diffusivity" and the lifetime respectively. The solution of (3) with the initial condition (2) is :

$$
n(x, t)=N e^{-t / \tau\left(1+\frac{16 \log 2}{a^{2}} \times D t\right)^{-\frac{1}{2}} \exp -\left\{x^{2} /\left[\left(a^{2} / 4 \log 2\right)+4 D t\right]\right\}}
$$

The luminescence detected in the $Y$ direction (Fig. 2) for a given wavelength $\lambda_{0}$ is a function of $x$. We call $I_{\lambda_{0}}\left(x, t_{i}\right)$ the distribution function for spontaneous luminescence intensity at time $t$, with $\lambda_{0}$ given by $\frac{h c}{\lambda_{0}}-E_{G}^{\prime}=\operatorname{constant}(=11 \mathrm{meV})$. For a phonon $L O$ assisted plasma luminescence, we have [2]:

$I_{\lambda}\left(x, t_{i}\right) \propto \int_{0}^{h \nu+k \omega_{L 0}-E_{G}^{\prime}} d \varepsilon_{c} \sqrt{\varepsilon_{c}} \sqrt{h \nu+h \omega_{L O}-E_{G}^{\prime}-\varepsilon_{c}}\left|M_{e l-p h}\right|^{2}\left(1+N_{q}\right) f_{e}\left(\varepsilon_{c}\right) f_{h}\left(h \nu+\hbar \omega_{10}-E_{G}^{\prime}-\varepsilon_{c}\right)$ where $M_{\text {el-ph }}$ is the matrix element for the electron-phonon interaction, $\varepsilon_{c}$ is the kinetic energy of the electron, $f_{e}$ and $f_{h}$ are the Fermi statistic factors for electrons and holes respectively, $N_{q}$ is the L0-phonon occupation number and $\hbar \omega_{L O}$ is the Lo-phonon energy.

We shall give latter an estimate of the plasma temperature, but in al1 cases, despite its high density, the hot plasma is rather close a non degenerate state (because the higher the excitation is, the higher the density is, but the higher the plasma temperature is !). If $N_{q}$ is lower than unity, the formula (5) gives :

(6) $I_{\lambda_{0}}\left(x, t_{i}\right) \propto \exp \mu_{k T}-\mu_{h} \propto n p \simeq n^{2}$ where $\mu_{e}$ and $\mu_{h}$ are the chemical potential for electrons and holes respectively, $T$ is the plasma temperature, and $k$ is the Boltzmann constant. 
With (6) and (2) we obtain :

$$
I_{\lambda_{0}}\left(x, t_{i}\right)=C\left[n\left(x, t_{i}\right)\right]^{2}=A\left(t_{i}\right) \exp -\frac{4 \log 2}{b_{i}^{2}} x^{2}
$$

where $i=1$ or 2 and $A\left(t_{j}\right)$ and $C$ are independent of $x$.

$b_{i}$ is defined by $b_{i}^{2} / 4 \log 2=\left(a^{2} / 8 \log 2\right)+2 D t_{i}$

Through the slit (Fig. 2), the total measured intensity distribution for $\lambda_{0}$ is :

$$
\begin{aligned}
& \Phi_{\lambda_{0}}\left(X, t_{i}\right)=K_{1} \int_{X-f / 2}^{X+8 / 2} I_{\lambda_{0}}\left(x, t_{i}\right) d x= \\
& K_{2}\left\{P\left[\frac{\sqrt{8 \log 2}}{b i}\left(X+\frac{f}{2}\right)\right]-P\left[\frac{\sqrt{8 \log 2}}{b_{i}}\left(X-\frac{f}{2}\right)\right]\right\}
\end{aligned}
$$

Here $K_{1}$ and $K_{2}$ are constants, $X$ is the distance between the centre of the excited zone and the centre of the slit (Fig. 2), $f$ is the slit width and $P(z)$ is the tabulated function :

$P(z)=\frac{1}{\sqrt{2 \pi}} \int_{-\infty}^{z} \exp -\left(u^{2} / 2\right) d u$. The best fit of the experimental data (Fig. 4) is obtained for $b_{1}=187 \mu \mathrm{m}, b_{2}=230 \mu \mathrm{m}$ at $t_{1}$ and $t_{2}$ respectively and $\tau \simeq 160 \mathrm{ps}$. We deduce $D \simeq 10^{6} \mathrm{~cm}^{\frac{1}{2}} / \mathrm{s}$. This value of $160 \mathrm{ps}$ for the parameter $\tau$ cannot be associated with the spontaneous luminescence on $7 y$, because the "diffusion" along $x$ is affected by the stimulation of light in this direction. The value for $D$ is more than four order of magnitude greater than the conventionnal diffusivity: the electron hole plasma blows up !

3. Discussion. - The mean kinetic energy of the photoexcited electron-hole pairs is here $\left(2 \mathrm{~h} \nu-E_{G}^{\prime}\right) \simeq 0.5 \mathrm{eV}$. When the density is high enough to permit the internal thermalisation of the plasma, i.e. $n \geqslant 10^{17} \mathrm{~cm}^{-3}$ [2], a temperature can be defined; we have calculated the temperature kinetics, but also the time dependent nonequilibrium distributions for the mixed plasmon-LO phonon modes [7] and for TO phonons modes.

Four consequences follow from the use of this analysis for our experimental results : - Firstly, we can verify the previous affirmation of non degenerate plasma statistics in the first 80 ps ( $p l$ asma temperature is always greater than $70 \mathrm{~K}$ ).

- Secondly, the knowledge of the plasma temperature allows us to estimate the thermodynamic equilibrium diffusivity, using the Einstein relation. The calculation gives a value for $D_{0}$ at least four orders of magnitude lower than our experimental values of $D$;

- Thirdly, we can calculate the absorption coefficient (which is a function of the plasma temperature) and see that the photon transfert [8] cannot be considered as the main mechanism in this rapid displacement of the luminescent plasma. 0therwise, the absence of hot luminescence in the experimental spectra supports this interpretation; - Fourthly, the part played by the non-equilibrium distributions of phonons is very important in the cooling of high density electron hole plasma. Moreover, at low lattice temperature, the plasma expands out of the initialiy excited region in a lattice with few phonons (except the ones created by plasma cooling) whereas at high lattice temperature $\left(T_{L} \sim 300 \mathrm{~K}\right)$ it does not expand.

M. Combescot [9] calculated the velocity for the expansion of a very dense plasma at 
$T=0 \mathrm{~K}$, using an hydrodynamic model ; if we use this model here, we obtain a value which is the tenth of our experimentally deduced result $V=\sqrt{\frac{D}{\tau}} \simeq 0.810^{8} \mathrm{~cm} / \mathrm{s}$.

$R$. Zimmermannand $M$. Rös ler calculated the free energy per pair $F$ in $C d S$ at different temperatures as a function of density [10]. We can calculate the pressure in the plasma using $p=n\left(\frac{\partial F}{\partial \log n}\right)_{T, N}$ where $N$ is the total number of electron-hole pairs; when the density is increased above the electron-hole liquid density

$\left(n_{0} \sim 5.4 \times 10^{17} \mathrm{~cm}^{-3}\right.$ in CdSe) this pressure rises up rapidly. This fact is in good agreement with a plasma expansion.

Conclusion. - We have studied the spatial expansion of the luminescent electron hole plasma at high density in CdSe, using a time, space and wavelength resolved picosecond photoluminescence experiment. The main result is that above a critical density, the plasma blow up at a velocity at least two orders of magnitude greater than the thermodynamic equilibrium value. A model for plasma explosion at $T \neq 0$ taking into account the non equilibrium distribution of phonons would be very complex; we think that the progress in this field depends mainly on the future experiments in various materials to approach the space resolved kinetics of the plasma temperature and non equilibrium distributions of phonons.

Referencès. -

1 M. Pugnet, A. Cornet, J. Collet, M. Brousseau, B.S. Razbirin, G.V. Michailov, Solid State Comm., 36, 85 (1980)

2 M. Pugnet, Thèse, Univ. Paul Sabatier, Toulouse (1981)

3 J. Collet, M. Pugnet, A. Cornet, M. Brousseau, B.S. Razbirin, G.V. Michailov, Phys. Status Solidi, 103, 367 (1981)

4 J.H. Betchel and W.L. Smith, Phys. Rev., 13, 3515 (1976)

5 N. Volovik and M. Strashnikova, Sov. Phys., Solid State, 20, 94 (1978)

6 E.0. Göbel , 0. Hi ldebrand and K. Löhnert, I.E.E.E. Journal of Quantum Electronics, vol. QE-13, no 10 (Oct. 1977)

7 J. Collet, A. Cornet, M. Pugnet, T. Amand, to be published

8 R. Bichard, Thèse, Paris VI (1979)

9 M. Combescot, Solid State Comm., 30, 81 (1979)

10 R. Zimmermann and M. Rösler, Phys. Stat. Sol. (b), 75, 633 (1976). 